\title{
DIVISION XII / COMMISSION 46 / PROGRAM GROUP EXCHANGE OF ASTRONOMERS
}

\author{
CHAIR \\ VICE-CHAIR \\ PAST CHAIR
}

\author{
John R. Percy \\ Kam-Ching Leung \\ Charles R. Tolbert
}

\section{TRIENNIAL REPORT 2006 - 2009}

\section{Introduction}

The Commission 46 Program Group Exchange of Astronomers (PG-EA) provides travel grants to astronomers and advanced students for research or study trips of at least three months duration. Highest priority is given to applicants from developing countries whose visits will benefit them, their institution and country, and the institution visited. This program, if used strategically, has the potential to support other Commission 46 programs such as Teaching for Astronomical Development (PG-TAD) and World Wide Development of Astronomy (PG-WWDA). Complete information about the program, and the application procedure, can be found at <physics.open.ac.uk/IAU46/travel.html>.

\section{Awarded grants}

From January 2006 to July 2008, 15 grants were awarded. Recipients came from: Brazil (1), China (1), Egypt (6), Honduras (2), Nigeria (2), Peru (1), Sweden (1), Uzbekistan (1). The visits were for a variety of useful purposes, including beginning a graduate program or post-doctoral fellowship, visiting a vibrant research centre such as International Centre for Theoretical Physics (Trieste) or Inter-University Centre for Astronomy and Astrophysics (Pune), visiting an institute to learn new techniques useful for research and education in the home country, and taking up a summer research assistantship.

\section{Improving the program}

We note, as previous reports of this PG have done, that the number of applications has been decreasing, despite the fact that the program is well-publicized, both on the IAU web page, and through the Organizing Committee of Commission 46. We also note that, with some exceptions, the grants do not directly support other IAU and Commission 46 development programs such as TAD and WWDA. The role of and rules for this PG should therefore be re-assessed. The rules could be broadened, while focussing the program more sharply on the IAU's development priorities. Indeed, a broad discussion and reorganization of IAU education and development activities is presently underway.

Acknowledgments: We thank the IAU General Secretary and the IAU Secretariat for their advice and assistance, and the host institutions and astronomers who have made these visits possible. 